\title{
Effectiveness of bariatric surgery in diabetes prevention in high-risk Asian individuals
}

\author{
Phong Ching Lee ${ }^{1}$, MBChB, FRCPEd, Hong Chang $\underline{\text { Tan }}^{1}$, MBBS, MRCP, Shanker Pasupathy ${ }^{2}$, MBBS, FRCS, \\ Sonali Ganguly ${ }^{1}$, MD, ABIM, Alvin Kim Hock Eng ${ }^{3}$, MBBS, FRCS, Nivedita Nadkarni ${ }^{4}$, PhD, Kwang Wei Tham $^{1}$, MD, ABIM
}

INTRODUCTION Obesity is a key risk factor in the development of Type 2 diabetes mellitus (T2DM). Bariatric surgery causes a large amount of durable weight loss in those with clinically severe obesity. We reported the effect of weight loss via bariatric surgery on DM prevention in those at high risk of developing DM.

METHODS This was a retrospective cohort study of 44 patients with obesity (mean body mass index $43.8 \mathrm{~kg} / \mathrm{m}^{2}$ ) and pre-DM who underwent bariatric surgery and were followed up for up to three years. We also reviewed a non-surgical cohort of patients with obesity and pre-DM seen at the weight management clinic.

RESULTS $91 \%$ of patients attained normal glycaemic status at one year after bariatric surgery. At the three-year followup, $87.5 \%$ of the patients maintained normoglycaemia. None of the patients developed T2DM after surgery. $26.9 \%$ of patients achieved absolute weight loss at one year after bariatric surgery and maintained this at two and three years post surgery ( $p<0.001$ vs. baseline). The homeostatic model assessment-insulin resistance index in patients also decreased from 5.50 at baseline to 1.20, 1.14 and 1.44 at one, two and three years, respectively $(p<0.001)$.

CONCLUSION Bariatric surgery produces significant weight loss, and leads to reversion from the pre-diabetic state to normal glycaemic status and reduction of the incident DM rate in those with pre-DM and morbid obesity.

Keywords: Asians, bariatric surgery, diabetes mellitus, diabetes prevention, obesity

\section{INTRODUCTION}

The global burden of the diabetes mellitus (DM) epidemic is well recognised, with increasing prevalence of DM and pre-DM in every region of the world. The number of people with Type 2 DM (T2DM) is projected to rise from 382 million in 2013 to 592 million by 2035. ${ }^{(1)}$ Obesity is a key risk factor in the development of T2DM. ${ }^{(2)}$ The global prevalence of overweight and obesity has risen by $27.5 \%$, from 857 million in 1980 to 2.1 billion in 2013..$^{(3)}$ The emerging pandemic in DM and obesity is an important public health issue, with a huge impact on patients, healthcare systems and societal costs. This underscores the importance of finding effective treatment strategies in the treatment and prevention of DM.

With any measure or surrogate of glycaemia, the risk of DM lies on a continuum, extending well into the normal ranges. Pre-DM broadly refers to an intermediate stage between completely normal glucose levels and the clinical entity of T2DM, encompassing both impaired fasting glucose (IFG) and impaired glucose tolerance (IGT). ${ }^{(4)}$ The average annual risk of developing T2DM in patients with IFG or IGT is $5 \%-10 \%$ per year, ${ }^{(5)} 5-10$ times that of individuals with normoglycaemia. ${ }^{(6)}$ Over a lifetime, the majority of patients with pre-DM will develop T2DM with no intervention.

Large, high-quality clinical trials ${ }^{(7-9)}$ showed that lifestyle modification via changes in diet and increasing physical activity can reduce the incidence of T2DM by $50 \%$ for people with pre-DM. In a landmark trial, the Diabetes Prevention Program aimed for weight reduction of at least $7 \%$ of initial body weight and increased physical activity in overweight or obese people at high risk of DM. ${ }^{(8)}$ The lifestyle intervention reduced the incidence of DM by $58 \%$ over an average follow-up duration of 2.8 years.

It is well established that T2DM improves with weight loss after bariatric surgery, with a recent systematic review showing that $66.7 \%$ of patients were able to attain DM remission after gastric bypass surgery. ${ }^{(9)}$ There are comparatively fewer studies examining the effect of surgery on DM prevention, consisting of one large observational study (Swedish Obese Subjects Study) $)^{(7)}$ and two smaller studies in the Caucasian population, ${ }^{(10,11)}$ all of which demonstrated reduced incidence of T2DM after surgery. At similar body mass index (BMI) levels, the prevalence of DM is higher in Asians compared with Caucasians. ${ }^{(12)}$ This may be due to the propensity in Asians to develop visceral adiposity, which is closely associated with insulin resistance and DM. ${ }^{(13)}$ Bariatric surgery and weight loss lead to improved insulin resistance, and the impact of bariatric surgery on DM prevention is unclear in the Asian cohort.

In this paper, we conducted a retrospective cohort study on patients with obesity and pre-DM, who were at high risk of developing DM, to examine the effect of bariatric surgery on T2DM prevention in the Asian population.

\section{METHODS}

Two groups of patients were included in the study - the surgery group and the conventional group. The surgery group had been diagnosed with pre-DM before they underwent bariatric surgery and were followed up for at least one year after surgery. Patients included for analysis underwent metabolic bariatric surgery from 
2009 to 2014 at Singapore General Hospital (SGH), Singapore. Criteria for surgery included $\mathrm{BMI} \geq 37.5 \mathrm{~kg} / \mathrm{m}^{2}$ or $\mathrm{BMI} \geq 32.5 \mathrm{~kg} / \mathrm{m}^{2}$ with any obesity-related complications. Follow-up data was reported on an annual basis for up to three years. Study data was collected and managed using REDCap (Research Electronic Data Capture) tools. ${ }^{(14)}$ This study was approved by the SingHealth Centralised Institutional Review Board.

Patients' weight, BMl and blood pressure were recorded at baseline and on follow-up. Reported biochemical measurements included glycated haemoglobin ( $\mathrm{HbA} 1 \mathrm{c})$, fasting plasma glucose, two-hour plasma glucose in the $75 \mathrm{~g}$ oral glucose tolerance test (OGTT) and lipid panel. Trained laboratory clinical staff were available on standby during OGTT, as incidents of reactive hypoglycaemia have been reported after OGTT in patients who had bariatric surgery. Fasting insulin levels were measured and the homeostatic model assessment-insulin resistance (HOMA-IR) index was calculated as a surrogate marker for insulin resistance, using the following equation: HOMA-IR = (fasting plasma insulin $\times$ fasting plasma glucose)/22.5..$^{(15)}$

We also reviewed a group of patients with pre-DM who attended the obesity management clinic at the Lifestyle Improvement and Fitness Enhancement Centre, SGH, and had at least one year of follow-up. These patients formed the conventional group. Patients at the clinic would generally be reviewed at regular intervals by a multidisciplinary team involving the physician, dietitian and exercise physiotherapist. They received individualised lifestyle modification advice, including one-to-one sessions with the dietitian as well as fitness assessment and exercise prescription by the exercise physiotherapist, and had access to supervised gym sessions at the centre.

The following criteria were used to define pre-DM: fasting plasma glucose of 5.6-6.9 mmol/L (100-125 mg/dL) and/or two-hour plasma glucose in the $75 \mathrm{~g}$ OGTT of $7.8-11.0 \mathrm{mmol} / \mathrm{L}$ (140-199 mg/dL) and/or HbA1c of 5.7\%-6.4\%. ${ }^{(16)}$ Glycaemic status was assessed for patients in both groups at baseline and annually for up to three years. We considered a patient to have T2DM if any of the following criteria were met: fasting plasma glucose $\geq 7.0 \mathrm{mmol} / \mathrm{L}$ (100-125 mg/dL); two-hour plasma glucose in the $75 \mathrm{~g}$ OGTT $\geq 11.1 \mathrm{mmol} / \mathrm{L}(140-199 \mathrm{mg} / \mathrm{dL}) ; \mathrm{HbA} 1 \mathrm{c} \geq 6.5 \%$.

Continuous variables were summarised as mean \pm standard deviation or median (interquartile range), as appropriate, and categorical variables as frequency (percentage). Two-sample $t$-tests or the Wilcoxon test were used, as appropriate, to compare the baseline characteristics between the surgery and the conventional group. A mixed effects model with a random effect for patient was used to assess the change in the outcome over time. The Wald test was used to test changes in the various outcome measures over time after bariatric surgery. All p-values were two-sided and a p-value $<0.05$ was considered statistically significant. Statistical analysis was performed using IBM SPSS Statistics version 21.0 (IBM Corp, Armonk, NY, USA) and R version 3.1.2 (R Foundation for Statistical Computing, Vienna, Austria).

\section{RESULTS}

A total of 69 patients with obesity and pre-DM were included in the study. Their mean age was 45.6 years and $73.9 \%$ of the patients were female. The study cohort was multi-ethnic (52.2\% Chinese, $21.7 \%$ Malay and $21.7 \%$ Indian). Baseline characteristics for the study cohort are summarised in Table I. There were 44 patients in the surgery group, of whom $34(77.3 \%)$ patients had laparoscopic sleeve gastrectomy (LSG), 9 (20.4\%) had laparoscopic gastric bypass (LGB) and 1 (2.3\%) had laparoscopic adjustable gastric banding. Their mean preoperative weight was $116.9 \pm 23.7 \mathrm{~kg}$ and mean BMI $43.8 \pm 6.5 \mathrm{~kg} / \mathrm{m}^{2}$. The conventional group consisted of 25 patients, with a mean BMl of $35.0 \pm 6.6 \mathrm{~kg} / \mathrm{m}^{2}$ at baseline. The conventional group was significantly older and had a significantly lower BMI compared with the surgery group.

Changes in weight and BMI after bariatric surgery are shown in Table II. There was significant weight loss of $31.4 \mathrm{~kg}$, from $116.9 \mathrm{~kg}$ at baseline to $85.5 \mathrm{~kg}$ at one year $(\mathrm{p}<0.001)$, resulting in an absolute weight loss of $26.9 \%$. BMI correspondingly decreased from $43.8 \mathrm{~kg} / \mathrm{m}^{2}$ at baseline to $32.0 \mathrm{~kg} / \mathrm{m}^{2}$ at one year $(\mathrm{p}<0.001)$. The weight loss was maintained with a mean body weight of $85.5 \mathrm{~kg}$ and $87.6 \mathrm{~kg}$ at two and three years, respectively.

Patients also showed improvement in markers of glycaemia after surgery. HbA1c levels decreased from $5.9 \%$ to $5.2 \%$ $(p<0.001)$ at one year, with significant improvement from baseline even at three years. Fasting plasma glucose levels decreased from $5.5 \mathrm{mmol} / \mathrm{L}$ at baseline to $4.7 \mathrm{mmol} / \mathrm{L}$ at one year $(p<0.001)$. Of those patients who had OGTT, 55\% of patients (12 out of 22 patients) developed hypoglycaemia at two hours after 75 g OGTT (0/1 patient who had LAGB, 8/15 patients who had LSG [53\%], 4/6 patients who had LGB [67\%]). HOMA-IR

Table I. Baseline characteristics of the study cohort $(n=69)$.

\begin{tabular}{|c|c|c|c|}
\hline \multirow[t]{2}{*}{ Variable } & \multicolumn{2}{|c|}{ No. (\%) } & \multirow[t]{2}{*}{ p-value } \\
\hline & $\begin{array}{l}\text { Surgery } \\
\text { group }(n=44)\end{array}$ & $\begin{array}{l}\text { Conventional } \\
\text { group }(n=25)\end{array}$ & \\
\hline Age $(y r) *$ & $43.2 \pm 11.4$ & $50.4 \pm 10.1$ & $<0.001$ \\
\hline Female gender & 29 (65.9) & $22(88.0)$ & $<0.050$ \\
\hline Ethnicity & & & 0.227 \\
\hline Chinese & $22(50.0)$ & $14(56.0)$ & \\
\hline Malay & $12(27.3)$ & $3(12.0)$ & \\
\hline Indian & $10(22.7)$ & $5(20.0)$ & \\
\hline Others & $0(0)$ & $3(12.0)$ & \\
\hline \multicolumn{4}{|l|}{ Type of surgery } \\
\hline LAGB & $1(2.3)$ & NA & \\
\hline LSG & $34(77.3)$ & NA & \\
\hline LGB & $9(20.4)$ & NA & \\
\hline Weight (kg)* & $116.9 \pm 23.7$ & $91.6 \pm 19.0$ & $<0.001$ \\
\hline BMI $\left(\mathbf{k g} / \mathbf{m}^{2}\right)^{*}$ & $43.8 \pm 6.5$ & $35.0 \pm 6.6$ & $<0.001$ \\
\hline HbA1c (\%)* & $5.9 \pm 0.3$ & $6.0 \pm 0.4$ & 0.596 \\
\hline FPG (mmol/L)* & $5.5 \pm 0.6$ & $6.3 \pm 0.6$ & $<0.001$ \\
\hline $\begin{array}{l}\text { 2-hr OGTT } \\
(\mathrm{mmol} / \mathrm{L}) *\end{array}$ & $8.0 \pm 1.4$ & $8.3 \pm 1.6$ & 0.602 \\
\hline SBP $(\mathrm{mmHg}) *$ & $125.1 \pm 16.8$ & $118.5 \pm 12.0$ & 0.205 \\
\hline DBP $(\mathrm{mmHg}) *$ & $80.5 \pm 13.5$ & $78.9 \pm 12.1$ & 0.732 \\
\hline
\end{tabular}

*Data presented as mean \pm standard deviation. BMI: body mass index; DBP: diastolic blood pressure; FPG: fasting plasma glucose; HbA1c: glycated haemoglobin; LAGB: laparoscopic adjustable gastric banding; LGB: laparoscopic gastric bypass; LSG: laparoscopic sleeve gastrectomy; NA: not applicable; OGTT: oral glucose tolerance test; SBP systolic blood pressure 
Table II. Clinical and metabolic parameters of patients at baseline and after bariatric surgery.

\begin{tabular}{|c|c|c|c|c|c|}
\hline \multirow[t]{2}{*}{ Parameter } & \multicolumn{4}{|c|}{ Mean \pm standard deviation } & \multirow[t]{2}{*}{ p-value* } \\
\hline & Baseline $(n=44)$ & $1 \mathrm{yr}(\mathrm{n}=44)$ & $2 \mathrm{yr}(\mathrm{n}=34)$ & 3 yr $(n=24)$ & \\
\hline Weight (kg) & $116.9 \pm 23.7$ & $85.5 \pm 16.5$ & $85.5 \pm 18.0$ & $87.6 \pm 14.2$ & $<0.001$ \\
\hline BMI $\left(\mathrm{kg} / \mathrm{m}^{2}\right)$ & $43.8 \pm 6.1$ & $32.0 \pm 5.1$ & $32.0 \pm 7.3$ & $32.1 \pm 4.9$ & $<0.001$ \\
\hline HbA1c (\%) & $5.9 \pm 0.3$ & $5.2 \pm 0.3$ & $5.3 \pm 0.3$ & $5.2 \pm 0.3$ & $<0.001$ \\
\hline FPG (mmol/L) & $5.5 \pm 0.6$ & $4.7 \pm 0.4$ & $4.9 \pm 0.4$ & $4.9 \pm 0.6$ & $<0.001$ \\
\hline 2-hr OGTT (mmol/L) & $8.0 \pm 1.4$ & $4.0 \pm 1.8$ & $3.3 \pm 0.6$ & $5.4 \pm 1.3$ & $<0.001$ \\
\hline HOMA-IR & $5.5 \pm 4.6$ & $1.2 \pm 0.8$ & $1.1 \pm 0.4$ & $1.4 \pm 0.6$ & $<0.001$ \\
\hline SBP (mmHg) & $125.1 \pm 16.8$ & $119.5 \pm 15.6$ & $121.9 \pm 14.9$ & $121.9 \pm 15.5$ & 0.120 \\
\hline $\mathrm{DBP}(\mathrm{mmHg})$ & $80.5 \pm 13.5$ & $73.6 \pm 10.5$ & $77.3 \pm 12.1$ & $77.7 \pm 13.0$ & 0.100 \\
\hline Antihypertensives (\%) & 60.0 & 21.4 & 22.2 & 9.1 & 0.005 \\
\hline Total cholesterol & $5.25 \pm 0.99$ & $5.09 \pm 0.80$ & $5.13 \pm 1.09$ & $5.37 \pm 1.35$ & 0.980 \\
\hline $\mathrm{HDL}$ & $1.16 \pm 0.28$ & $1.45 \pm 0.40$ & $1.49 \pm 0.29$ & $1.49 \pm 0.38$ & $<0.001$ \\
\hline Triglycerides & $1.37 \pm 0.42$ & $0.98 \pm 0.59$ & $0.89 \pm 0.32$ & $1.03 \pm 0.37$ & $<0.001$ \\
\hline LDL & $3.48 \pm 1.00$ & $3.16 \pm 0.76$ & $3.26 \pm 0.93$ & $3.41 \pm 1.12$ & 0.300 \\
\hline
\end{tabular}

*Wald test was used to determine statistical significance. BMI: body mass index; DBP: diastolic blood pressure; FPG: fasting plasma glucose; HbA1c: glycated haemoglobin; HDL; high-density lipoprotein; HOMA-IR: homeostatic model of assessment-insulin resistance index; LDL: low-density lipoprotein; OGTT: oral glucose tolerance test; SBP: systolic blood pressure

Table III. Weight change and glycaemic status of patients with up to three years of follow-up.

\begin{tabular}{|lllll|}
\hline Parameter & Baseline & $\mathbf{1}$ yr & $\mathbf{2}$ yr & $\mathbf{3} \mathbf{~ y r}$ \\
\hline Surgery group & $\mathrm{n}=44$ & $\mathrm{n}=44$ & $\mathrm{n}=34$ & $\mathrm{n}=24$ \\
\hline $\begin{array}{l}\text { Weight loss from } \\
\text { baseline (kg) }\end{array}$ & - & 31.4 & 31.4 & 29.3 \\
\hline $\begin{array}{l}\text { Absolute weight loss (\%) } \\
\text { Glycaemic status (no.) }\end{array}$ & & 26.9 & 26.9 & 25.1 \\
\hline$\quad$ Normal & 0 & 40 & 31 & 21 \\
\hline $\begin{array}{l}\text { Pre-diabetes mellitus } \\
\text { Type 2 diabetes mellitus }\end{array}$ & 0 & 4 & 3 & 3 \\
\hline Conventional group & $\mathrm{n}=25$ & $\mathrm{n}=21$ & $\mathrm{n}=21$ & $\mathrm{n}=18$ \\
\hline $\begin{array}{l}\text { Weight loss from } \\
\text { baseline (kg) }\end{array}$ & - & 4.4 & 3.3 & 1.5 \\
\hline Absolute weight loss (\%) & - & 4.8 & 3.6 & 1.6 \\
\hline Glycaemic status (no.) & & & & \\
\hline Normal & 0 & 3 & 5 & 7 \\
\hline Pre-diabetes mellitus & 25 & 16 & 9 & 3 \\
\hline Type 2 diabetes mellitus & 0 & 2 & 7 & 8 \\
\hline
\end{tabular}

values also decreased from 5.5 at baseline to $1.2,1.1$ and 1.4 at one, two and three years, respectively $(p<0.001)$. Blood pressure was similar after surgery, although the proportion of patients requiring antihypertensive medications declined significantly ( $p=0.005$ ) (Table II). There was a significant increase in high-density lipoprotein levels $(p<0.001)$ and decrease in triglyceride levels $(p<0.001)$ at one, two and three years after surgery. Low-density lipoprotein levels decreased in the first and second year ( $p=0.022$ ), with subsequent non-statistically significant increases in the second and third year $(p=0.30$ and 0.24 , respectively).

Among the cohort of pre-DM patients who underwent bariatric surgery, 91\% attained normal glycaemic status at one year after surgery. The number of patients with a longer duration of postoperative follow-up was smaller. For those who had two- and three-year follow-up data, most maintained normoglycaemia. Notably, none of the patients developed T2DM (Table III).
In the conventional group, the incidence of T2DM in the high-risk obese pre-diabetic cohort was 9.5\%, 33.3\% and 44.4\% at one, two and three years, respectively. A significant proportion managed to achieve normal glycaemic status, with $38.9 \%$ attaining normoglycaemia at three years. There was significant weight loss of $4.4 \mathrm{~kg}$ in the first year $(p=0.02)$. Weight was regained in the second and third year such that by three years, the weight change was not statistically significant $(p=0.490)$ The patients who attained normoglycaemia at three years had mean weight loss of $3 \mathrm{~kg}$, while those who progressed to develop T2DM gained $1 \mathrm{~kg}$ on average.

\section{DISCUSSION}

The vast majority of patients with pre-DM reverted to normal glycaemic status after bariatric surgery, and normoglycaemia was sustained for up to three years after surgery. The striking effect of surgical intervention could be due to the higher residual beta-cell function of the study population. T2DM is a progressive disease, with decline in beta-cell function over time. ${ }^{(17)}$ It is estimated that approximately $50 \%$ of beta-cell function remains at the point of diagnosis of DM. ${ }^{(18)}$ Hence, improvements in insulin sensitivity from weight loss after surgery may not be enough to result in DM remission if the disease is already advanced. Indeed, the duration of DM is inversely related to the DM remission rate after bariatric surgery. ${ }^{(19)}$ Higher C-peptide levels, a marker of beta-cell reserve, are also a predictive factor for DM remission after surgery. ${ }^{(20)}$ In our previous report on bariatric surgery in T2DM patients, we found that $53 \%$ of patients were able to achieve complete DM remission after surgery, ${ }^{(21)}$ suggesting that early and aggressive intervention for morbid obesity and pre-DM via bariatric surgery is effective in modifying the natural history of progressive beta-cell dysfunction in individuals with pre-DM. Improvements in HOMA-IR suggested that the mechanism is due to decreased insulin resistance from weight loss. Other glycaemic markers, including $\mathrm{HbA} 1 \mathrm{c}$ and OGTT results, were significantly better at one year after surgery, with sustained decrease in $\mathrm{HbA} 1 \mathrm{c}$ levels at three years. 
In the present study, patients in the conventional group who attended the weight management clinic managed to achieve mean weight loss of $4.4 \%$ at one year. The amount of weight loss was less than that in the DM prevention trials and similar to the results seen in community-based weight loss programmes. ${ }^{(22)}$ At three years' follow-up, divergence in glycaemic status was observed; $38.8 \%$ of the patients reverted to normoglycaemia, whereas $44.4 \%$ developed DM. The group that reverted to normoglycaemia lost weight, while the group that developed DM gained weight from the baseline. Other studies have shown a similar relationship between weight loss and DM prevention. Post-hoc analysis of the Diabetes Prevention Program showed that weight loss was by far the most important contributor in the prevention of DM.(23) Randomised trials have also showed that weight loss strongly associated with reduced DM risk. ${ }^{(24)}$ Our study similarly showed consistent findings. The reduced rate of incident DM in the surgery cohort compared with the conventional cohort mirrored the amount of weight loss observed. Thus, the focus on weight loss in lifestyle modification efforts appears to be crucial in DM prevention. ${ }^{(25)}$

In this study, patients from the conventional group were highly motivated individuals who were able to adhere to lifestyle advice, and the default rate was low even at three years. Given our inclusion criteria that patients must have at least one year of follow-up data, patients who defaulted or did not complete followup for at least a year were excluded from analysis. We expected that the rate of progression to T2DM would be even higher if the patients were unable to lose weight or less motivated to make the necessary lifestyle changes. Indeed, a range of hormonal changes occur following weight loss, which drives increased energy intake and reduced energy expenditure. These physiological adaptations limit the durability of weight loss and the beneficial effects that it offers. Bariatric surgery, on the other hand, leads to substantial and durable weight loss, and consequent metabolic benefits, including improvement in glycaemic control. However, it is not without risks and complications. Surgery would be an impractical choice for most individuals with pre-DM. In our cohort, most of the patients underwent surgery primarily for clinically severe obesity or other obesity-related complications.

Although landmark clinical trials have shown that we can delay or even prevent development of DM in high-risk individuals, it cannot be assumed that the results can be easily translated to clinical practice. Our report on the effect of bariatric surgery and conventional therapy in DM prevention in the real-world environment could hence add useful information about DM prevention in clinical practice. To our knowledge, this is the first study that examines bariatric surgery and DM prevention in Asians. The Asian experience is particularly important, given phenotypic differences between Asians and Caucasians in relation to DM.

However, this study had several limitations, including the retrospective design, single-centre site and short duration of follow-up. The number of patients who completed three years of follow-up was small, as a significant proportion of patients had their operations performed less than three years prior to the conduct of this study. Additionally, data on quality of life and complications following bariatric surgery was not available. The baseline clinical characteristics of the surgery and conventional groups were also different, and therefore a direct comparison could not be made between the two groups.

In conclusion, this study suggests that bariatric surgery is highly effective in delivering substantial and sustained weight loss over three years, hence preventing or delaying the development of DM in the high-risk Asian population with pre-DM and morbid obesity. Larger, longer-term studies are required to provide more information on the durability of the protective effect of surgery against DM and the cost-effectiveness of surgery in preventing DM as well as other obesity and DM-related complications.

\section{REFERENCES}

1. Guariguata L, Whiting DR, Hambleton I, Beagley J, Linnenkamp U, Shaw JE. Global estimates of diabetes prevalence for 2013 and projections for 2035. Diabetes Res Clin Pract 2014; 103:137-49.

2. Colditz GA, Willett WC, Rotnitzky A, Manson JE. Weight gain as a risk factor for clinical diabetes mellitus in women. Ann Intern Med 1995; 122:481-6.

3. $\mathrm{Ng} \mathrm{M}$, Fleming $\mathrm{T}$, Robinson $\mathrm{M}$, et al. Global, regional, and national prevalence of overweight and obesity in children and adults during 1980-2013: a systematic analysis for the Global Burden of Disease Study 2013. Lancet 2014; 384:766-81.

4. Aroda VR, Ratner R. Approach to the patient with prediabetes. J Clin Endocrinol Metab 2008; 93:3259-65.

5. Gerstein HC, Santaguida P, Raina $P$, et al. Annual incidence and relative risk of diabetes in people with various categories of dysglycemia: a systematic overview and meta-analysis of prospective studies. Diabetes Res Clin Pract 2007; 78 : 305-12.

6. Inzucchi SE. Clinical practice. Diagnosis of diabetes. N Engl J Med 2012; 367:542-50.

7. Carlsson LM, Peltonen M, Ahlin S, et al. Bariatric surgery and prevention of type 2 diabetes in Swedish obese subjects. N Engl J Med 2012; 367:695-704.

8. Knowler WC, Barrett-Connor E, Fowler SE, et al. Reduction in the incidence of type 2 diabetes with lifestyle intervention or metformin. N Engl J Med 2002; 346:393-403.

9. Puzziferri N, Roshek TB 3rd, Mayo HG, et al. Long-term follow-up after bariatric surgery: a systematic review. JAMA 2014; 312:934-42.

10. Long SD, O'Brien K, MacDonald KG Jr, et al. Weight loss in severely obese subjects prevents the progression of impaired glucose tolerance to type II diabetes. A longitudinal interventional study. Diabetes Care 1994; 17:372-5.

11. Pontiroli AE, Folli F, Paganelli M, et al. Laparoscopic gastric banding prevents type 2 diabetes and arterial hypertension and induces their remission in morbid obesity: a 4-year case-controlled study. Diabetes Care 2005; 28:2703-9.

12. King GL, McNeely MJ, Thorpe LE, et al. Understanding and addressing unique needs of diabetes in Asian Americans, native Hawaiians, and Pacific Islanders. Diabetes Care 2012; 35:1181-8.

13. Araneta MR, Barrett-Connor E. Ethnic differences in visceral adipose tissue and type 2 diabetes: Filipino, African-American, and white women. Obes Res 2005; 13:1458-65.

14. Harris PA, Taylor R, Thielke R, et al. Research electronic data capture (REDCap)--a metadata-driven methodology and workflow process for providing translational research informatics support. J Biomed Inform 2009; 42:377-81.

15. Matthews DR, Hosker JP, Rudenski AS, et al. Homeostasis model assessment: insulin resistance and beta-cell function from fasting plasma glucose and insulin concentrations in man. Diabetologia 1985; 28:412-9.

16. American Diabetes Association. (2) Classification and diagnosis of diabetes. Diabetes Care 2015; 38 Suppl:S8-S16.

17. Marchetti P, Dotta F, Lauro D, Purrello F. An overview of pancreatic beta-cell defects in human type 2 diabetes: implications for treatment. Regul Pept 2008; 146:4-11.

18. Wajchenberg BL. beta-cell failure in diabetes and preservation by clinical treatment. Endocr Rev 2007; 28:187-218.

19. Hall TC, Pellen MG, Sedman PC, Jain PK. Preoperative factors predicting remission of type 2 diabetes mellitus after Roux-en-Y gastric bypass surgery for obesity. Obes Surg 2010; 20:1245-50.

20. Lee MH, Lee WJ, Chong K, et al. Predictors of long-term diabetes remission after metabolic surgery. J Gastrointest Surg 2015; 19:1015-21.

21. Lee PC, Tham KW, Tan HC, Pasupathy S. Impact of bariatric surgery on the management of type 2 diabetes mellitus in Singapore. Singapore Med J 2013; 54:382-6.

22. Kahn R, Davidson MB. The reality of type 2 diabetes prevention. Diabetes Care 2014; 37:943-9.

23. Hamman RF, Wing RR, Edelstein SL, et al. Effect of weight loss with lifestyle intervention on risk of diabetes. Diabetes Care 2006; 29:2102-7.

24. Heymsfield SB, Segal KR, Hauptman J, et al. Effects of weight loss with orlistat on glucose tolerance and progression to type 2 diabetes in obese adults. Arch Intern Med 2000; 160:1321-6.

25. Ratner RE, Sathasivam A. Treatment recommendations for prediabetes. Med Clin North Am 2011; 95:385-95, viii-ix. 